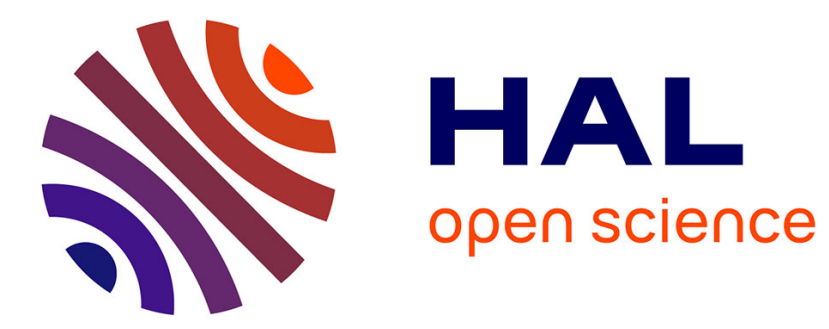

\title{
Incorporation of a deformation prior in image reconstruction
}

\author{
Barbara Gris
}

\section{To cite this version:}

Barbara Gris. Incorporation of a deformation prior in image reconstruction. Journal of Mathematical Imaging and Vision, 2019. hal-01810443

\section{HAL Id: hal-01810443 \\ https://hal.science/hal-01810443}

Submitted on 7 Jun 2018

HAL is a multi-disciplinary open access archive for the deposit and dissemination of scientific research documents, whether they are published or not. The documents may come from teaching and research institutions in France or abroad, or from public or private research centers.
L'archive ouverte pluridisciplinaire HAL, est destinée au dépôt et à la diffusion de documents scientifiques de niveau recherche, publiés ou non, émanant des établissements d'enseignement et de recherche français ou étrangers, des laboratoires publics ou privés. 


\title{
Incorporation of a deformation prior in image reconstruction

\author{
Barbara Gris *
}

\begin{abstract}
This article presents a method to incorporate a deformation prior in image reconstruction via the formalism of deformation modules. The framework of deformation modules allows to build diffeomorphic deformations that satisfy a given structure. The idea is to register a template image against the indirectly observed data via a modular deformation, incorporating this way the deformation prior in the reconstruction method. We show that this is a well-defined regularization method (proving existence, stability and convergence) and present numerical examples of reconstruction from 2-D tomographic simulations and partially-observed images.
\end{abstract}

\section{Introduction}

For many imaging techniques, the acquisition time is relatively long. For instance in computed tomography targetting the torso, the acquisition takes several minutes and then the patient breathes during the acquisition. Using static reconstruction methods leads then to the appearance of motion artefacts which can prevent from identifying some structures or, on the contrary, creates false ones. The solution that is used in clinic for torso computed tomography is to use "gated data": the respiratory rythm of the patient is recorded simultaneously, and only the data acquired at a specific respiratory state are used for the reconstruction. In order to be able to use all the available data, it is necessary to incorporate a temporal component in the reconstruction method [17, 19, 20, 26].

In order to do so, an common strategy $[1,7,9,10,11,15,16,18,21,24,25,30]$ is to reconstruct one initial image $I_{0}$ and a trajectory of deformations $t \mapsto \varphi_{t}$ such that a each time $t$ the image $\phi_{t} \cdot I_{0}$ (deformation of $I_{0}$ by $\phi_{t}$ ) matches the observed data. Then the framework has two intertwined components, estimation of $I_{0}$ and estimation of $t \mapsto \varphi_{t}$, that can be alternatively performed in an iterative optimization scheme. This article concentrates on the second step : estimating the deformation trajectory $t \mapsto \varphi_{t}$, given observed data and an initial template image $I_{0}$. A central point is to define the deformation model, i.e. the set of deformations that are considered and their parametrization. In [10] and [16] for instance, the deformation model is built via the LDDMM framework [6], leading to good numerical and theoretical results. However, as illustrated in the following, this deformation model corresponds to unstructured deformations in the sense that it is not possible to incorporate a prior knowledge about the type of deformations that can occur. As a consequence, in some cases the estimated deformation is not intuitively satisfying but there is no possibility with such unstructured-deformation framework to enforce a more intuitive solution. Several frameworks allow to incorporate particular prior in deformation models $[5,4,12,22,23,27,28,29,31]$ so that they are adapted to specific situations. The goal of this article is to show how a generic prior on the set of deformations can be incorporated via the notion of deformation modules [14] so that

\footnotetext{
${ }^{*}$ LJLL - Laboratoire Jacques-Louis Lions, UPMC, Paris, France.
} 
only the desired solutions are used to reconstruct an image from the observed data and the initial template. For instance in the case of biological images, this framework would ensure that only the deformations that are possible from a biological point of view are considered. The interest of the deformation module framework is that it encompasses many previous approaches and requires very few conditions on the constraints that can be incorporated in the deformation model.

We recall the notion of deformation modules and build a particular class of deformation modules called constrained translations generator deformation modules that can be easily built and used. We present how geodesic trajectories can be used to reconstruct an image from indirect observations and a given initial template, and then we show that this strategy is a well-defined regularization method to solve inverse problems by proving the existence of solutions as well as their stability and convergence. Finally we present several numerical examples, using our framework to reconstruct images from 2-D simulations of two different natures: tomographic data (obtained via the 2-D Radon transform) and partial observation (obtained by restricting the image on a small window).

\section{Background}

\subsection{Inverse problem}

Let $\Omega \subset \mathbb{R}^{d}$ be a fixed bounded domain and $X:=L^{2}(\Omega, \mathbb{R})$ be a space of grey scale images on $\Omega$. The principle of inverse problem resolution is to reconstruct an image $I$ belonging to the space $X$ from an indirect observation $g$ belonging to a data space $Y$. More precisely, we suppose that there is a ground truth image $I_{\text {truth }}$ in $X$ and an operator $T: X \mapsto Y$ such that the observed data is $g=T\left(I_{\text {truth }}\right)+\epsilon$ where $\epsilon$ is some noise. The goal is to build an image $I$ such that $T(I) \simeq g$ and that is satisfying from a certain point of view to be defined (for example regularity).

\subsection{Large deformations and indirect registration}

The idea behind indirect registration as developed for instance in $[10,16,18]$, is to search the image to reconstruct as the deformation of a known template image $I_{0}$ by a deformation $\varphi$. It is then necessary to define how deformations can transform an image. There are several possible choices, in the following we will consider the geometric group action of diffeomorphisms on $X$ defined by $\varphi \cdot I=I \circ \varphi^{-1}$ for $\varphi \in \operatorname{Diff}_{0}^{\ell}(\Omega)$ and $I \in X$.

The deformation that we will consider are large deformations defined as flows of a time-varying vector-field :

Proposition 1. [2] Let $V$ be a fixed Hilbert space of vector fields on $\mathbb{R}^{d}$ continuously embedded in $C_{0}^{\ell}(\Omega)$ (vector fields $\ell$ times continuously differentiable, supported on $\Omega$, with derivatives tending to zero at the boundary) and let $v \in E^{2}([0,1], V)$. Then the following equation

$$
\left\{\begin{array}{l}
\frac{\mathrm{d}}{\mathrm{d} t} \varphi_{t}(x)=\nu\left(t, \varphi_{t}(x)\right) \\
\varphi_{t=0}=I d
\end{array} \quad \text { for any } x \in \Omega \text { and } t \in[0,1] .\right.
$$

has a unique absolutely continuous solution and it is a diffeomorphism at each time. It is called the flow of $v$ and we will denote it by $\varphi_{t}^{v} \in \operatorname{Diff}_{0}^{\ell}\left(\mathbb{R}^{d}\right)$. 


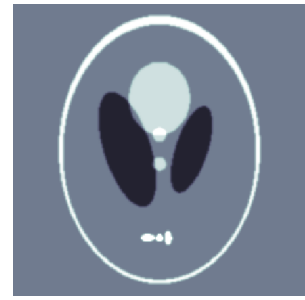

(a) Template.

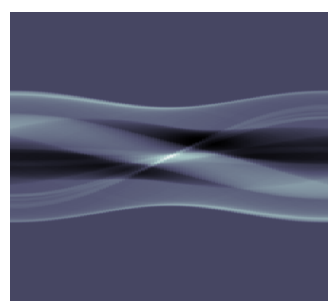

(b) Data.

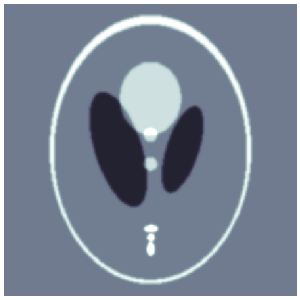

(c) Ground truth.

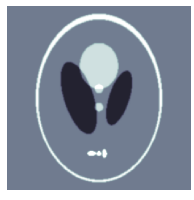

(d) $t=0$.

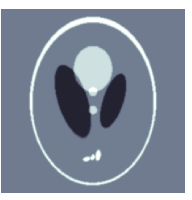

(e) $t=0.25$.

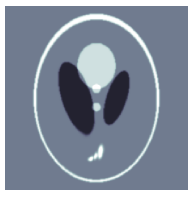

(f) $t=0.5$.

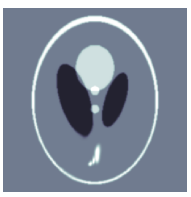

(g) $t=0.75$.

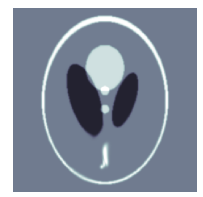

(h) $t=1$.

Figure 1: Result of LDDMM-based indirect matching (without constraint). Template $I_{0}$ in fig. 1a matched against data $g$ in fig. 1b obtained from ground truth in fig. 1c (forward operator : Ray transform with 100 angles uniformly distributed 0 and $\pi$ ). Second row : image trajectory $\varphi_{t}^{v} \cdot I_{0}$, the reconstructed image is in fig. $1 \mathrm{~h}$.

In this context, the strategy of indirect registration of a template image $I_{0} \in X$ against some data $g \in Y$ is then to minimize a functional of the form

$$
J: v \in \mathrm{E}^{2}([0,1], V) \mapsto C(v)+\frac{1}{\lambda} D\left(T\left(\varphi_{t=1}^{v} \cdot I_{0}\right), g\right)
$$

where $D$ is a distance on $Y$.

This framework leads to good result (see [10, 16]) but sometimes the obtained deformation, and then the reconstructed image, are not intuitively satisfying. For instance we present in Figure 1 the result of the indirect registration of the template image presented in Figure 1a against the data $g$ presented in Figure 1b which are the Ray transform with 100 angles uniformly distributed 0 and $\pi$ of the ground truth image Figure 1c. Even though the reconstructed image in Figure $1 \mathrm{~h}$ is not too far (for the $L^{2}$ metric for instance) from the ground truth image, intuitively it would have been more satisfying to obtain a deformation rotating the small white structure than one distorting it like here. It would be interesting to force the deformation to be a local rotation, and then to optimise the parameters of this rotation. However, with this non structured indirect matching, it is not possible to incorporate the additional knowledge of the type of transformation that we would like to observe.

\section{Deformation modules}

The object of this article is to show how the framework of deformation modules introduced in [14] can be used to incorporate motion prior in image reconstruction.

\subsection{Definition}

The intuition behind the deformation module framework is to constrain deformations in order to incorporate some prior in the motion, while leaving some parameters free in order 
to be able to adapt to data. For instance if the goal is to reconstruct a respiratory motion, even though this motion is different from one patient to another, there might be some shared "base-motions" from which any respiratory motion can be reconstructed. These "base-motions" can be modelled by some generators that, given the current "geometrical state" of the subject, would define a family of vector fields which can then be combined to produce the respiratory motion. The current "geometrical state" of the subject can be given via its image or some other geometrical variable such as landmarks, and the coefficients of the combination of the vector fields correspond to a "control variable" in the sense that they have to be optimized so that the global motion fits to the data. The framework of deformation modules formalizes this intuition. The idea of "geometrical state" is formalized by the notion of "shape" as defined by S. Arguillere in [3]:

Definition 1. Let $\mathcal{O}$ be a manifold of finite dimension and $k \in \mathbb{N}^{*}$. Assume that the group Diff ${ }_{0}^{\ell}\left(\mathbb{R}^{d}\right)$ acts continuously on $\mathcal{O}$, according to the action

$$
\begin{aligned}
\operatorname{Diff}_{0}^{\ell}\left(\mathbb{R}^{d}\right) \times \mathcal{O} & \rightarrow \mathcal{O} \\
(\phi, o) & \mapsto \phi \cdot o .
\end{aligned}
$$

We say that $\mathcal{O}$ is a $C^{k}$-shape space of order $\ell$ on $\mathbb{R}^{d}$ if the following conditions are satisfied:

1. For each $o \in \mathcal{O}, \phi \in \operatorname{Diff}_{0}^{\ell}\left(\mathbb{R}^{d}\right) \mapsto \phi \cdot o$ is Lipschitz with respect to the norm $|\cdot|_{\ell}$ and is differentiable at $I d_{\mathbb{R}^{d}}$. This differential is called the infinitesimal action of $C_{0}^{\ell}\left(\mathbb{R}^{d}\right)$ and we will simply denote the action of a vector field $v$ on a geometrical descriptor o (with a slight abuse of notation) $v \cdot o$.

2. The mapping $(o, v) \in \mathcal{O} \times C_{0}^{\ell}\left(\mathbb{R}^{d}\right) \mapsto v \cdot o$ is continuous and its restriction to $\mathcal{O} \times$ $C_{0}^{\ell+k}\left(\mathbb{R}^{d}\right)$ is of class $C^{k}$.

An element o of $\mathcal{O}$ is called a shape, and $\mathbb{R}^{d}$ will be referred to as the ambient space.

We now give a slightly simplified formal definition of a deformation module from the one defined in [14]:

Definition 2. Let $k, \ell \in \mathbb{N}^{*}$. We say that $M=(\mathcal{O}, H, \zeta, c)$ is a $C^{k}$-deformation module of order $\ell$ with geometrical descriptors in $\mathcal{O}$, controls in $H$, field generator $\zeta$ and cost $c$, if

- $\mathcal{O}$ is a $C^{k}$-shape space of $\mathbb{R}^{d}$ of order $\ell$ with an infinitesimal action $C_{0}^{\ell}\left(\mathbb{R}^{d}\right) \times \mathcal{O} \longrightarrow$ $T \mathcal{O}$,

- $H$ is a finite-dimensional Euclidean space,

- $\zeta:(o, h) \in \mathcal{O} \times H \rightarrow\left(o, \zeta_{o}(h)\right) \in \mathcal{O} \times C_{0}^{\ell}\left(\mathbb{R}^{d}\right)$ is continuous, with $h \mapsto \zeta_{o}(h)$ linear and $o \mapsto \zeta_{o}$ of class $C^{k}$,

- $c:(o, h) \in \mathcal{O} \times H \rightarrow c_{o}(h) \in \mathbb{R}^{+}$is a continuous mapping such that $o \mapsto c_{o}$ is smooth and for all $o \in \mathcal{O}, h \mapsto c_{o}(h)$ is a positive quadratic form on $H$, thus defining a smooth metric on $\mathcal{O} \times H$.

The field generator $\zeta$ plays the role of generator of the "base-motions", it takes in input couples of a geometrical descriptor and a control variable. The geometrical descriptor is the variable giving some geometrical information and leading to the specification of the constraints (for instance specifying the location of the generated vector field). The 
control variable specifies how to combine these constraints. As the geometrical descriptor corresponds to "geometric information", if the geometry of the ambient space is modified through a deformation, the geometrical descriptor should be transformed accordingly. This is why it is necessary to specify how vector fields can act on geometrical descriptors via the infinitesimal action of the shape space $\mathcal{O}$. The importance of this parameter will be detailed with the definition of modular large deformations in Section 3.4.

Remark 1. In [14], the deformation module was defined by a five-fold $(\mathcal{O}, H, \zeta, \xi, c)$ where $\xi$ is the infinitesimal action associated to the shape space $\mathcal{O}$. Here in order to simplify the notations (and as in the examples we present there is no ambiguity about them ), we will denote all the infinitesimal actions $v \cdot o$ and they will be implicitly defined via the shape spaces of geometrical descriptors.

In the following we will restrict ourselves to deformation modules satisfying the Uniform Embedding Condition:

Definition 3. Let $M=(\mathcal{O}, H, \zeta, c)$ be a $C^{k}$-deformation module of order $\ell$. We say that $M$ satisfies the Uniform Embedding Condition (UEC) if there exists a Hilbert space of vector fields $V$ continuously embedded in $C_{0}^{\ell+k}\left(\mathbb{R}^{d}\right)$ and a constant $C>0$ such that for all $o \in \mathcal{O}$ and for all $h \in H, \zeta_{o}(h) \in V$ and

$$
\left|\zeta_{o}(h)\right|_{V}^{2} \leq C c_{o}(h) .
$$

This condition will be required for the theoretical results presented in the following sections.

\subsection{Examples}

We will now present some examples that are very simple to define and that will simultaneously be very useful in the following. They all satisfy the uniform embedding condition. All the images are defined on $\Omega=[-16,16] \times[-16,16]$ which is discretized in $256 \times 256$ pixels.

\subsubsection{Local translations}

Let us consider again the image in Figure 1a and imagine that there is a prior on the way it can be transformed. Suppose that we know that there are two forces that can push or pull in any direction, acting in areas of given sizes. A way to model these forces is by using local translations. Then let us build a deformation module generating vector fields that are always a sum of two local translations, localized via a Gaussian kernel $K_{\sigma}:(x, y) \mapsto \exp -\frac{|x-y|^{2}}{2 \sigma^{2}}$ (we fix the kernel size $\sigma$ ). The generated vector fields will then be parametrized by :

- 2 points, centres of the local translations : they define the locations of the translations given the current geometrical state and then are geometrical descriptors

- 2 vectors, vectors of the local translations : they define how the two local translations can be used to generate an adapted vector field and then they are control variables.

The space of geometrical descriptors is therefore $\mathcal{O}=\mathbb{R}^{2} \times \mathbb{R}^{2}$ (space of two points), the space of controls is $H=\mathbb{R}^{2} \times \mathbb{R}^{2}$ (space of two vectors) and the field generator is $\zeta:(o, h) \in \mathcal{O} \times H \mapsto \sum_{i=1}^{2} K_{\sigma}\left(o_{i}, \cdot\right) h_{i}$ with $o=\left(o_{1}, o_{2}\right)$ and $h=\left(h_{1}, h_{2}\right)$. A natural choice for the infinitesimal action of $\mathcal{O}$ is the application of vector fields to the two points: 

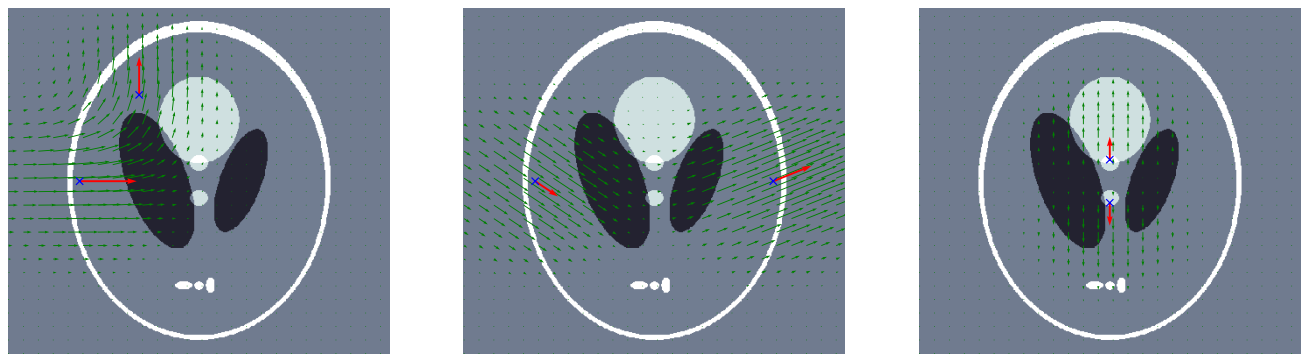

Figure 2: Three examples of vector field generated by a deformation module generating sums of two local translations (see Section 3.2.1, $\sigma=6$ ) for three different values of geometrical descriptors and controls. The blue crosses are the geometrical descriptors, the red arrows are the controls and the vector fields are plotted in green.

$(o, v) \in \mathcal{O} \times C_{0}^{\ell}\left(\Omega, \mathbb{R}^{2}\right) \mapsto v \cdot o \doteq\left(v\left(o_{1}\right), v\left(o_{2}\right)\right)$ with $o=\left(o_{1}, o_{2}\right)$. The cost can be chosen as $c:(o, h) \mapsto\left|\zeta_{o}(h)\right|_{V_{\sigma}}^{2}$ with $V_{\sigma}$ the RKHS associated with $K_{\sigma}$ so that the defined deformation module $M=(\mathcal{O}, H, \zeta, c)$ straightforwardly satisfies the UEC. The set of vector fields that can be generated by this deformation module is rich, as illustrated in Figure 2, but these vector fields follow the strong prior of being sums of two local translations.

\subsubsection{Contracting-dilating field}

Suppose now that one has an additional prior on the directions of the vectors of the two translations: that they should both be parallel to the line between the two centres and in opposite direction. In this case it is not adapted to let the vectors of the translation being controls variables as they cannot be chosen freely. On the contrary the directions are now a function of the geometrical descriptor, and the variable that can now be freely chosen is a scalar to which will be multiplied the vectors of the translations. More precisely we can set, for this new deformation module, $\tilde{\mathcal{O}}=\mathbb{R}^{2} \times \mathbb{R}^{2}, \tilde{H}=\mathbb{R}$ and $\tilde{\zeta}:(o, h) \in \tilde{\mathcal{O}} \times \tilde{H} \mapsto$ $h\left(K\left(o_{1}, \cdot\right)-K\left(o_{2}, \cdot\right)\right)\left(o_{1}-o_{2}\right)$ with $o=\left(o_{1}, o_{2}\right)$. We define as previously, the infinitesimal action of $\tilde{\mathcal{O}}$ by $(o, v) \in \tilde{\mathcal{O}} \times C_{0}^{\ell}\left(\Omega, \mathbb{R}^{2}\right) \mapsto v \cdot o \doteq\left(v\left(o_{1}\right), v\left(o_{2}\right)\right)$ and $\tilde{c}:(o, h) \mapsto\left|\zeta_{o}(h)\right|_{V_{\sigma}}^{2}$. We present in Figure 3 several examples of vector fields generated by this deformation module $\tilde{M}=(\tilde{\mathcal{O}}, \tilde{H}, \tilde{\zeta}, \tilde{c})$. Note that the vector fields generated by $\tilde{M}$ can also be generated by $M$ but that they are not parametrized in the same manner : an additional prior comes with $\tilde{M}$.

\subsubsection{Constrained translations generator deformation modules}

In the following we will use a certain category of deformation module that generate vector fields which are a constrained sum of local translations, generalizing the ones previously presented in Section 3.2.2. More precisely we set a scale $\sigma \in \mathbb{R}^{+} *, N \in \mathbb{N}$ and two functions $f:\left(\mathbb{R}^{d}\right)^{N} \mapsto\left(\mathbb{R}^{d}\right)^{p}$ (a point-generator function) and $g:\left(\mathbb{R}^{d}\right)^{N} \mapsto\left(\mathbb{R}^{d}\right)^{p}$ (a vector-generator function) with $p \in \mathbb{N}$. Then we define $\mathcal{O}=\left(\mathbb{R}^{d}\right)^{N}$ (space of $N$ points), $H=\mathbb{R}$ and $\zeta:(o, h) \in \mathcal{O} \times H \mapsto h \sum_{i=1}^{p} K_{\sigma}\left(f_{i}(o), \cdot\right) g_{i}(o)$ with $f=\left(f_{i}\right)$ and $g=\left(g_{i}\right)$. The idea here is to associate, to each geometrical descriptor $o$, a set of points $\left(f_{1}(o), \cdots, f_{p}(o)\right)$ and a set of vectors $\left(g_{1}(o), \cdots, g_{p}(o)\right)$ so that the vector fields that can be generated with $o$ are colinear to the sum of the local translations centred at points $f_{i}(o)$ with vectors $g_{i}(o)$. The infinitesimal action can be simply defined by the application of the vector field to the points composing the geometrical descriptor and the cost by $c:(o, h) \in \mathcal{O} \times H \mapsto C\left|\zeta_{o}(h)\right|_{V_{\sigma}}^{2}+h^{2}$ 

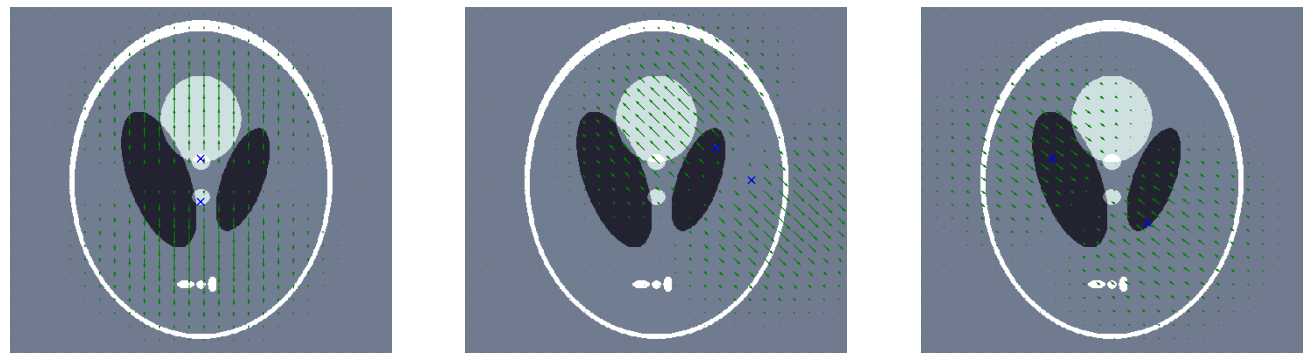

Figure 3: Three examples of vector field generated by a deformation module generating contracting or dilating field (see Section 3.2.2, $\sigma=8$ ) for three different values of geometrical descriptors and controls. The blue crosses are the geometrical descriptors, the vector fields are plotted in green. The scalar control is positive for the left and middle figure, and negative for the figure on the right.

for some $C>0$. This definition as a sum of these two terms is due to regularity reasons, ensuring that $c_{o}$ is a quadratic form on $H$ for all $o$ in $\mathcal{O}$ and that the deformation module satisfies the uniform embedding condition. Deformation modules that can be defined this way will be called constrained translations generator deformation modules.

These deformation modules are defined by three parameters : the kernel-size $\sigma$, the point-generator function $f$ and the vector-generator function $g$. We present in Figure 4 various vector fields generated by various deformation modules, i.e. for various choices of $\sigma, f$ and $g$.

\subsection{Combining deformation modules}

An interesting feature of this framework is that deformation modules can be combined to form a compound deformation module that will generate vector fields that are a sum of the vector fields generated by the combined deformation modules. More precisely :

Definition 4. Let $M^{l}=\left(\mathcal{O}^{l}, H^{l}, \zeta^{l}, c^{l}\right), l=1 \cdots L$, be $L C^{k}$-deformation modules of order $\ell$. We define the compound module of modules $M^{l}$ by $\mathcal{C}\left(M^{l}, l=1 \cdots L\right)=(\mathcal{O}, H, \zeta, \xi, c)$ where $\mathcal{O} \doteq \prod_{l} \mathcal{O}^{l}, H \doteq \prod_{l} H^{l}$ and for $o=\left(o^{l}\right)_{l} \in \mathcal{O}, \zeta_{o}: h=\left(h^{l}\right) \in H \mapsto \sum_{l} \zeta_{o^{l}}^{l}\left(h^{l}\right)$, $v \cdot o=\left(v \cdot o^{l}\right)_{l} \in T_{o} \mathcal{O}\left(\right.$ for $v \in C_{0}^{\ell}\left(\mathbb{R}^{d}\right)$ ) and $c_{o}: h=\left(h^{l}\right) \in H \mapsto \sum_{l} c_{o^{l}}^{l}\left(h^{l}\right)$.

As shown in [14], the uniform embedding condition is stable result under combination and then an easy way to build complex deformation modules satisfying the uniform embedding condition is to combine several simple deformation modules satisfying this condition.

In Figure 5 we present three examples of vector fields generated by two different compound deformation modules.

\subsection{Modular large deformations}

The structure of deformation modules allows to constrain vector fields, we will now present how this can be used to incoporate a structure in large deformations. These large deformations are obtained as flow of time-varying vector fields and the idea is then to consider only vector fields that can be generated by the field generator of a given deformation modules. These trajectories of vector fields are then parametrized by trajectories of geometrical descriptors and controls and then in order to defined modular large deformations, one needs 

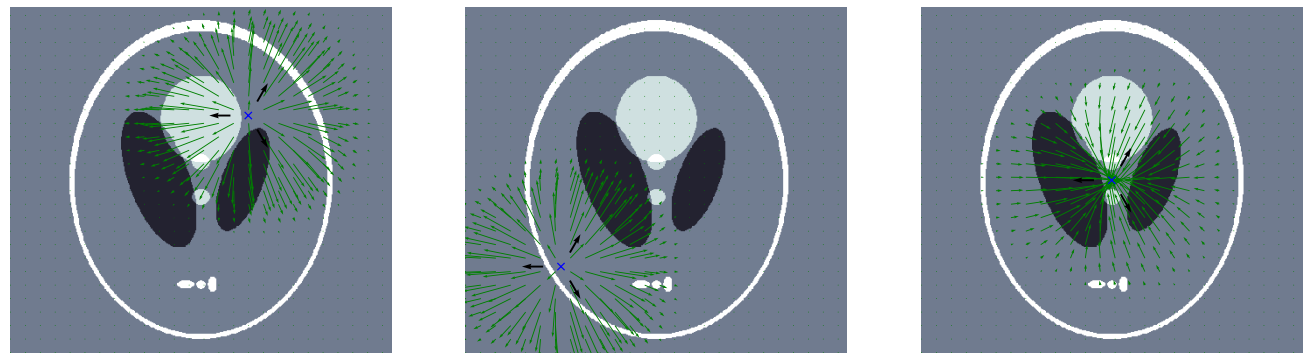

(a) Local scaling, $\sigma=5$
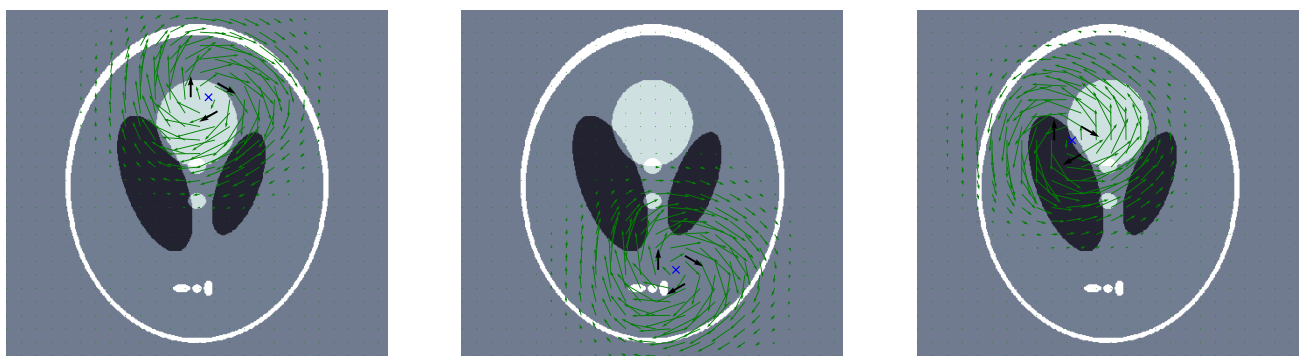

(b) Local rotation, $\sigma=5$
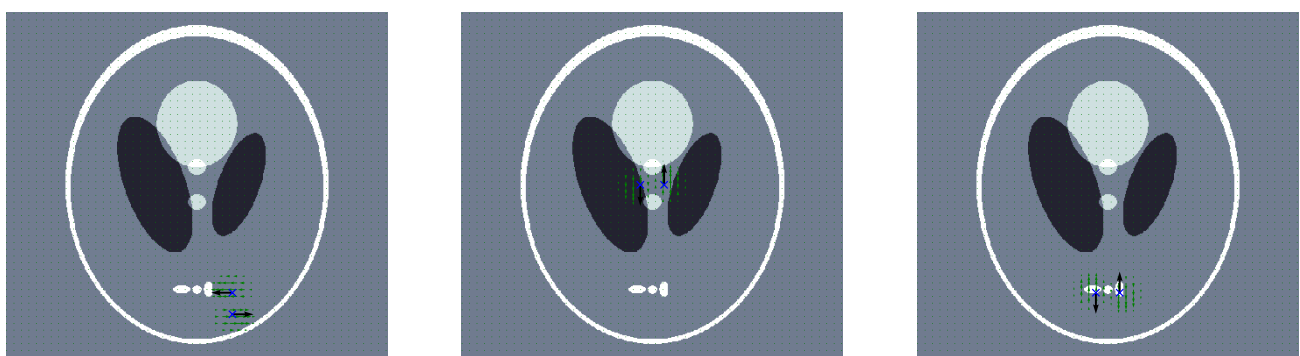

(c) Local shearing, $\sigma=1.5$

Figure 4: Examples of vector fields generated by three constrained translations generator deformation modules (see Section 3.2.3) for three different choices of kernel-size $\sigma$, point-generator function $f$ and vector-generator function $g$, leading to three types of vector fields: local scaling (fig. 4a), local rotation (fig. 4b) and local shearing (fig. 4c). For each deformation module, we present 3 examples of generated vector field for three different values of geometrical descriptors and controls. The blue crosses are the geometrical descriptors, vectors generated by the vector-generator functions $g$ are in black (their basepoints are points defined by $f(o)$ ) and the vector fields are plotted in green (the scalar controls are not represented, they are positive for left and middle figures, and negative for the right one). 

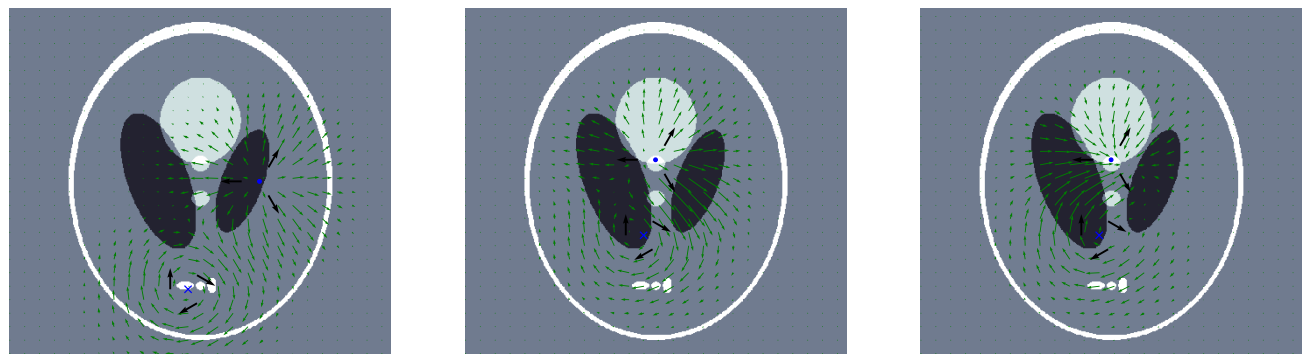

(a) Vector fields generated by combining a local rotation $(\sigma=5$, geometrical descriptors are blue crosses) and a local scaling ( $\sigma=5$, geometrical descriptors are blue dots)
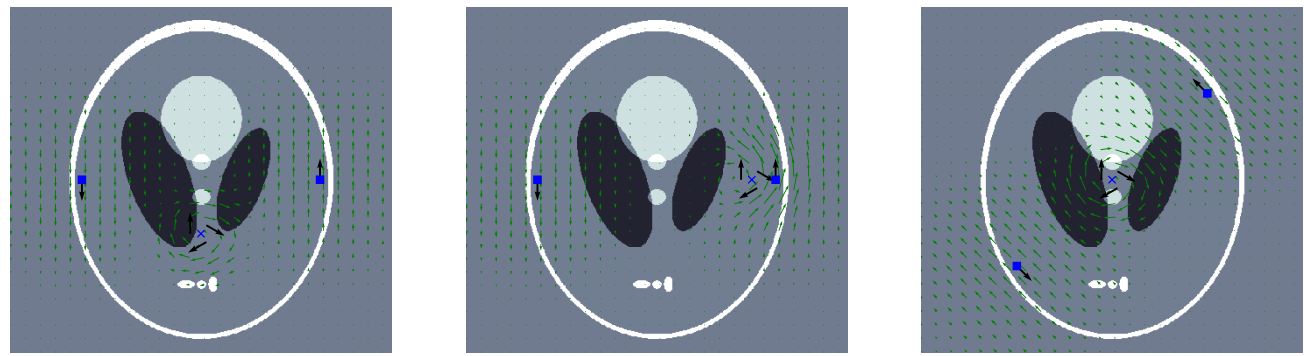

(b) Vector fields generated by combining a local rotation $(\sigma=5$, geometrical descriptors are blue crosses) and a local shearing ( $\sigma=8$, geometrical descriptors are blue squares)

Figure 5: Examples of vector fields generated by two compound deformation modules. In fig. 5a are represented vector fields generated by combining deformation modules generating local scaling and local rotations. In fig. 5b are represented vector fields generated by combining deformation modules generating local shearing and local rotations. For each of the two compound deformation modules, we present 3 examples of generated vector field for three different values of geometrical descriptors and controls. The geometrical descriptors are plotted in blue, vectors generated by the vector-generator functions $g$ are in black (their base-points are points defined by $f(o)$ ) and the vector fields in green (the scalar controls are not represented). 
to specify the trajectories of geometrical descriptors and controls that will be considered. We will consider what we call controlled path of finite energy:

Definition 5. Let $M=(\mathcal{O}, H, \zeta, c)$ be a deformation module and let $a, b$ be in $\mathcal{O}$. We denote $\Omega_{a, b}$ the set of measurable curves $t \mapsto\left(o_{t}, h_{t}\right) \in \mathcal{O} \times H$ where ot is absolutely continuous (a.c.), starting from $a$ and ending at $b$, such that for almost every $t \in[0,1]$, $\dot{o}_{t}=v_{t} \cdot o_{t}$, where $v_{t} \doteq \zeta_{o_{t}}\left(h_{t}\right)$, and

$$
E(o, h) \doteq \int_{0}^{1} c_{o t}\left(h_{t}\right) \mathrm{d} t<\infty .
$$

The quantity $E(o, h)$ is called the energy of $(o, h)$ and $\Omega_{a, b}$ is the set of controlled paths of finite energy starting at $a$ and ending at $b$.

If the UEC is satisfied, it is shown in [14] that large deformations can be built from controlled paths of finite energy :

Proposition 2. Let us suppose that $M$ satisfies UEC. Let $(o, h) \in \Omega_{a, b}$ and for each $t, v_{t}=\zeta_{o t}\left(h_{t}\right)$. Then $v \in L^{2}([0,1], V) \subset L^{1}$, the flow $\varphi^{v}$ exists, $h \in L^{2}([0,1], H)$ and for each $t \in[0,1], o_{t}=\phi_{t}^{v} . o_{0}$. We call the final diffeomorphism $\varphi_{t=1}^{v}$ a modular large deformation generated by a.

In Figure 6 we present an example of modular large deformation generated by the combination of two deformation modules. The first one generates "shearing" field at the scale $\sigma=8$ its geometrical descriptors are formed of two points, the point-generator function is $f: o \mapsto o$ (identical function) and the vector-generator function is $g: o=$ $\left(o_{1}, o_{2}\right) \mapsto\left(\left(o_{1}-o_{2}\right)^{T},\left(o_{2}-o_{1}\right)^{T}\right)$ with $x^{T} \doteq\left(x_{2},-x_{1}\right)$ for $x=\left(x_{1}, x_{2}\right) \in \mathbb{R}^{2}$ (vectors of $g(o)$ are orthogonal to the line between the two points of $o$ ). The second deformation module generates local rotations (at the scale $\sigma=3$ ). This trajectory is obtained with constant positive controls.

This example illustrates that geometrical descriptors naturally follow the deformation of the ambient space during modular large trajectories due to the equation $\dot{o}_{t}=\zeta_{o t}\left(h_{t}\right) \cdot o_{t}$. We emphasize here that the geometrical descriptors of the two combined deformation modules are transported by the total vector field generated by the compound deformation module: in particular the centre of the rotation is displaced by the shearing field. Then, the area which is both rotated and translated by the shearing motion remains the same during the whole trajectory. Note that this is a direct consequence of the definition of the combination of deformation modules and that in order to build such deformations, one only needs to define two deformation modules and then apply the simple combination rule defined in Section 3.3.

\subsection{Shooting equations}

Let $M=(\mathcal{O}, H, \zeta, c)$ be a combination of constrained translations generator deformation module. As shown in [14], normal geodesics (minimizing energy $E$ ) starting at $a \in \mathcal{O}$ can be parametrized by an initial variable called the initial momentum $\eta_{0} \in T_{a}^{*} \mathcal{O}$. The geodesic $(o, h)$ parametrized by $\left(a, \eta_{0}\right) \in T^{*} \mathcal{O}$ is given by : $o_{t=0}=a, \eta_{t=0}=\eta_{0}$ and

$$
\left\{\begin{aligned}
\frac{\mathrm{d} o_{t}}{\mathrm{~d} t} & =\zeta_{o t}\left(h_{t}\right) \cdot o_{t} \\
\frac{\mathrm{d} \eta_{t}}{\mathrm{~d} t} & =-\partial_{o} H\left(o_{t}, \eta_{t}, h_{t}\right) \\
h & =C_{o}^{-1}\left(\xi_{o} \circ \zeta_{o}\right)^{*}(\eta)
\end{aligned}\right.
$$

where $H:(o, \eta, h) \in T^{*} \mathcal{O} \times H \mapsto\left(\eta, \zeta_{o}(h) \cdot o\right)-\frac{1}{2} c_{o}(h)$ is the hamiltonian of the system and for each $o$ in $\mathcal{O} C_{o}: H \mapsto H^{*}$ is defined by $\left(C_{o} h, h\right)_{H^{*}, H}=c_{o}(h)$ for all $h$ in $H$. 


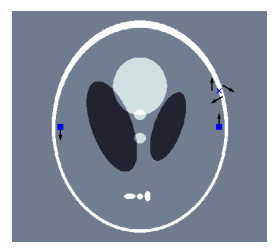

(a) $t=0$

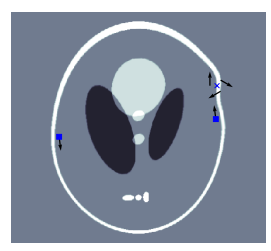

(b) $t=0.25$

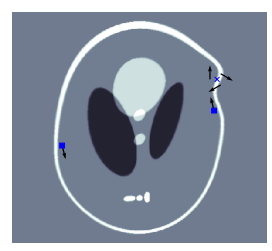

(c) $t=0.5$

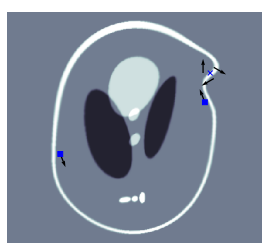

(d) $t=0.75$

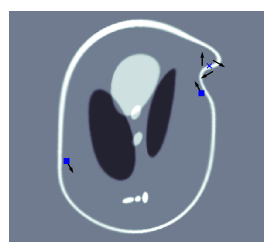

(e) $t=1$

Figure 6: Example of large deformation generated by the combination of two deformation modules. Geometrical descriptors are in blue (squares for shearing deformation module and cross for the rotation one) and vectors generated by the vector-generator functions $g$ are in black.

Lemma 1. If $M=(\mathcal{O}, H, \zeta, c)$ is a combination of $N$ constrained translations generator deformation modules (see Section 3.2.3), then for each o in $\mathcal{O}$, the operator $C_{o}: H \mapsto H^{*}$ defined by $\left(C_{o} h, h\right)_{H^{*}, H}=c_{o}(h)$ for all $h$ in $H$ is invertible and $C^{-1}: o \in \mathcal{O} \mapsto C_{o}^{-1}$ is smooth.

Proof. Let us denote $M^{k}=\left(\mathcal{O}^{k}, H^{k}, \zeta^{k}, c^{k}\right), k=1, \ldots, N$ the constrained translations generator deformation modules of which $M$ is the combination. For each $k$, there exist functions $f_{i}^{k}$ and $g_{i}^{k}, i=1, \ldots, p_{k}$ such that $\zeta^{k}$ is given by $\zeta^{k}:(o, h) \in \mathcal{O} \times H \mapsto$ $h \sum_{i=1}^{p_{k}} K_{\sigma}\left(f_{i}^{k}(o), \dot{)} g_{i}^{k}(o)\right.$. From the definition of the cost one gets that for each $k$ and for all $(o, h)$ in $\mathcal{O}^{k} \times H^{k}, C_{o}^{k}(h)=h\left(1+\sum_{i, j} K_{\sigma}\left(f_{i}^{k}(o), f_{i^{\prime}}^{k}(o)\right) g_{i}^{k}(o)^{T} g_{i}^{k}(o)\right)$ (let us remaining that the control $h \in H^{k}$ is scalar). Since $K_{\sigma}$ is a reproducing kernel, the quantity $\sum_{i, j} K_{\sigma}\left(f_{i}^{k}(o), f_{i^{\prime}}^{k}(o)\right) g_{i}^{k}(o)^{T} g_{i}^{k}(o)$ is always non-negative. As a consequence, the operator $C_{o}^{k^{-1}}$ is well defined for all $o$ in $\mathcal{O}^{k}$ and from the smoothness of functions $f_{i}^{k}$ and $g_{i}^{k}$ on gets that $o \in \mathcal{O}^{k} \mapsto C_{o}^{k^{-1}}=\frac{1}{1+\sum_{i, j} K_{\sigma}\left(f_{i}^{k}(o), f_{i^{\prime}}^{k}(o)\right) g_{i}^{k}(o)^{T} g_{i}^{k}(o)}$ is smooth.

This is true for all $k$ and as $C_{o}$ is defined by $C_{o}: h=\left(h_{1}, \ldots, h_{N}\right) \in H \mapsto\left(C_{o_{1}}^{1}\left(h_{1}\right), \ldots, C_{o_{N}}^{N}\left(h_{N}\right)\right)$ for all $o=\left(o_{1}, \ldots, o_{N}\right)$ in $\mathcal{O}$ (this is a direct consequence of the definition of the cost of a compound deformation module, see Section 3.3), it is clearly invertible and $o \mapsto C_{o}^{-1}$ is smooth.

\section{Image reconstruction with a deformation prior}

\subsection{Framework}

The idea here is, given a deformation module $M=(\mathcal{O}, H, \zeta, c)$, to search amongst all geodesics parametrized by an initial variable in $T^{*} \mathcal{O}$ the one allowing to perform the indirect registration. We will only consider deformation modules of the form described in Section 3.2.3 so that $\mathcal{O}=\Omega^{N}$ for some $N \in \mathbb{N}$ and $T^{*} \mathcal{O}=\Omega^{N} \times\left(\mathbb{R}^{d}\right)^{N}$. Let $I_{0}$ be a template image and $g$ be some data, the modular indirect registration will correspond to minimizing :

$$
J_{I_{0}, g, T}:\left(a, \eta_{0}\right) \in T^{*} \mathcal{O} \mapsto \gamma C_{1}(a)+\tau C_{2}(\eta)+D\left(T\left(\varphi_{t=1}^{\zeta_{o}(h)} \cdot I_{0}\right), g\right)
$$

where $(o, \eta)$ starts at $\left(o_{t=0}, \eta_{t=0}\right)=\left(a, \eta_{0}\right)$ and satisfies Equation $(3), \gamma, \tau \in \mathbb{R}^{+} *$ and $C_{1}: \mathcal{O} \mapsto \mathbb{R}^{+}, C_{2}:\left(\mathbb{R}^{d}\right)^{N} \mapsto \mathbb{R}^{+}$are continuous and satisfy $C_{1}(o) \longrightarrow+\infty$ when $o \longrightarrow \partial \Omega^{N}, C_{2}(\eta) \longrightarrow+\infty$ when $|\eta| \longrightarrow+\infty$. 


\subsection{Regularising properties}

Proposition 3 (Existence). Let $M=(\mathcal{O}, H, \zeta, c)$ be a constrained translations generator deformation module (see section 3.2.3), $I_{0} \in L^{2}(\Omega, \mathbb{R})$ a template image, $T: L^{2}(\Omega, \mathbb{R}) \mapsto Y$ a continuous operator with $Y$ a Banach space, and let $g$ be some data in $Y$. Then there $J_{I_{0}, g, T}$ has a minimizer in $T^{*} \mathcal{O}$.

Proof. Because of the conditions on the regularization functions $C_{1}$ and $C_{2}$ and the fact that $T^{*} \mathcal{O}$ is of finite dimension, it is sufficient to check that $J_{I_{0}, g, T}$ is continuous. First, from the smoothness of the Hamiltonian, it is easy to see that the trajectory $\left(o^{a, \eta_{0}}, \eta^{a, \eta_{0}}\right)$ satisfying Equation (3) is a continuous function of the initial conditions $\left(a, \eta_{0}\right)$ in $C\left([0,1], T^{*} \mathcal{O}\right)$ associated with the supremum norm. Then the trajectory of the optimal control $h^{a, \eta_{0}}$ is also a continuous function of $\left(a, \eta_{0}\right)$ in $C([0,1], H)$ associated with the supremum norm and the same result is true for the trajectory of vector field $\zeta\left(o, h^{a, \eta_{0}}\right)$. As a consequence (see [8]), $\left(a, \eta_{0}\right) \mapsto \varphi_{t=1}^{\zeta\left(a^{a, \eta_{0}}, h^{a, \eta_{0}}\right)} \cdot I_{0} \in L^{2}(\Omega, \mathbb{R})$ is a continuous function which concludes the proof.

Proposition 4 (Stability). Let $M=(\mathcal{O}, H, \zeta, c)$ be a constrained translations generator deformation module (see section 3.2.3), $I_{0} \in L^{2}(\Omega, \mathbb{R})$ a template image, $T: L^{2}(\Omega, \mathbb{R}) \mapsto Y$ a continuous operator with $Y$ a Banach space, and let $g^{k}$ be sequence of $Y$ that converges to $g \in Y$. For each $k$, let $\left(a^{k}, \eta_{0}^{k}\right)$ be a minimizer of $J_{I_{0}, g^{k}, T}$. Then there exists a sub-sequence of $\left(a^{k}, \eta_{0}^{k}\right)$ that converges to a minimizer of $J_{I_{0}, g, T}$.

Proof. Let $a \in \mathcal{O}$, for each $k, J_{I_{0}, g^{k}, T}\left(a^{k}, \eta_{k}^{0}\right) \leq J_{I_{0}, g^{k}, T}(a, 0)=C_{1}(a)+C_{2}(0)+\frac{1}{\lambda} D\left(T\left(I_{0}\right), g_{k}\right) \longrightarrow$ $C_{1}(a)+C_{2}(0)+\frac{1}{\lambda} D\left(T\left(I_{0}\right), g\right)$. Then the sequences $C_{1}\left(a^{k}\right)$ and $C_{2}\left(\eta_{0}^{k}\right)$ are bounded and as a consequence $\left(a^{k}, \eta_{0}^{k}\right)$ is in a compact set of $T^{*} \mathcal{O}$ (because it is of finite dimension). Then up to an extraction, we can suppose that $\left(a^{k}, \eta_{0}^{k}\right)$ converges to $\left(a^{\infty}, \eta_{0}^{\infty}\right)$ which leads to $J_{I_{0}, g^{k}, T}\left(a^{k}, \eta_{0}^{k}\right) \longrightarrow J_{I_{0}, g, T}\left(a^{\infty}, \eta_{0}^{\infty}\right)$ (because $g^{k} \longrightarrow g$ ). Then let $\left(a, \eta_{0}\right)$ be in $\mathcal{O}^{*}$, for each $k, J_{I_{0}, g^{k}, T}\left(a^{k}, \eta_{0}^{k}\right) \leq J_{I_{0}, g^{k}, T}\left(a, \eta_{0}\right)$ so when taking the limit of both terms one gets $J_{I_{0}, g, T}\left(a^{\infty}, \eta_{0}^{\infty}\right) \leq J_{I_{0}, g, T}\left(a, \eta_{0}\right)$. This is true for any $\left(a, \eta_{0}\right)$ so $\left(a^{\infty}, \eta_{0}^{\infty}\right)$ is a minimizer of $J_{I_{0}, g, T}$.

Proposition 5 (Convergence). Let $M=(\mathcal{O}, H, \zeta, c)$ be a constrained translations generator deformation module (see section 3.2.3), $I_{0} \in L^{2}(\Omega, \mathbb{R})$ a template image, $T$ : $L^{2}(\Omega, \mathbb{R}) \mapsto Y$ a continuous operator with $Y$ a Banach space, and let $g \in Y$. Assume that there exists $\left(\hat{a}, \hat{\eta}_{0}\right) \in T^{*} \mathcal{O}$ such that $T\left(\varphi_{t=1}^{\zeta^{\hat{a}, \hat{\eta}_{0}}} \cdot I_{0}\right)=g$. Furthermore, assume that there exists a parameter selection rule $\gamma: \mathbb{R}^{*}+\mapsto \mathbb{R}^{*}+, \tau: \mathbb{R}^{*}+\mapsto \mathbb{R}^{*}+$ such that $\delta \mapsto \gamma(\delta) / \tau(\delta)$ and $\delta \mapsto \tau(\delta) / \gamma(\delta)$ are bounded and $\gamma(\delta) \rightarrow 0, \tau(\gamma) \rightarrow 0, \delta^{2} / \gamma(\delta) \rightarrow 0$, $\delta^{2} / \tau(\delta) \rightarrow 0$ as $\delta \rightarrow 0$.

Let $\left(\delta_{k}\right)$ be a sequence in $\mathbb{R}^{*}+$ converging to 0 and let $\left(g_{k}\right)$ be a sequence in $Y$ such that $D\left(g_{k}, g\right) \leq \delta_{k}$ for each $k$. Finally let, for each $k,\left(a^{k}, \eta_{0}^{k}\right)$ be a minimizer of $J_{I_{0}, g^{k}, T}$. Then there exists a sub-sequence of $\left(a^{k}, \eta_{0}^{k}\right)$ that converges to a minimizer of $J_{I_{0}, g, T}$.

Proof. We set for each $k, \gamma_{k}=\gamma\left(\delta_{k}\right)$ and $\tau_{k}=\tau\left(\delta_{k}\right)$. Then, for each $k$ we have :

$$
\begin{aligned}
C_{1}\left(a^{k}\right) & \leq \frac{1}{\gamma_{k}} J_{I_{0}, g^{k}, T}\left(a^{k}, \eta_{0}^{k}\right) \leq \frac{1}{\gamma_{k}} J_{I_{0}, g^{k}, T}\left(\hat{a}, \hat{\eta}_{0}\right) \\
& =\frac{1}{\gamma_{k}}\left(\gamma_{k} C_{1}(\hat{a})+\tau_{k} C_{2}\left(\hat{\eta}_{0}\right)+D\left(T\left(\varphi_{t=1}^{\hat{a}, \hat{\eta}_{0}} \cdot I_{0}\right), g_{k}\right)\right) \\
& \leq C_{1}(\hat{a})+\frac{\tau_{k}}{\gamma_{k}} C_{2}\left(\hat{\eta}_{0}\right)+\frac{\delta_{k}^{2}}{\gamma_{k}}
\end{aligned}
$$

From the hypothesis, we deduce that $C_{1}\left(a^{k}\right)$ is bounded and then that $a_{k}$ is in a compact set. In a similar way we can show that $\eta_{0}^{k}$ is in a compact set so up to an extraction we can suppose that $\left(a^{k}, \eta_{0}^{k}\right)$ converges to $\left(a^{\infty}, \eta_{0}^{\infty}\right)$. As shown previously, this 
leads to $D\left(T\left(\varphi_{t=1}^{\zeta^{a^{k}, \eta_{0}^{k}}} \cdot I_{0}\right), g\right) \longrightarrow D\left(T\left(\varphi_{t=1}^{\zeta^{a^{\infty}, \eta_{0}^{\infty}}} \cdot I_{0}\right), g\right)$ and then, $D\left(T\left(\varphi_{t=1}^{\zeta^{a^{k}, \eta_{0}^{k}}} \cdot I_{0}\right), g^{k}\right) \leq$ $D\left(T\left(\varphi_{t=1}^{\zeta^{k}, \eta_{0}^{k}} \cdot I_{0}\right), g\right)+D\left(g, g_{k}\right) \longrightarrow D\left(T\left(\varphi_{t=1}^{\zeta^{\infty}, \eta_{0}^{\infty}} \cdot I_{0}\right), g\right)$. Besides, $D\left(T\left(\varphi_{t=1}^{\zeta^{a^{k}}, \eta_{0}^{k}} \cdot I_{0}\right), g^{k}\right) \leq$ $J_{I_{0}, g^{k}, T}\left(a_{k}, \eta_{0}^{k}\right) \leq J_{I_{0}, g^{k}, T}\left(\hat{a}, \hat{\eta}^{0}\right)=\gamma_{k} C_{1}(\hat{a})+\tau_{k} C_{2}\left(\hat{\eta}_{0}\right)+D\left(g, g_{k}\right) \longrightarrow 0$. As a consequence :

$$
D\left(T\left(\varphi_{t=1}^{\zeta^{\infty}, \eta_{0}^{\infty}} \cdot I_{0}\right), g\right)=0
$$

which concludes the proof.

\section{Application to image reconstruction}

\subsection{Overview}

We present here examples of image reconstruction via modular indirect matching. In order to do so we minimize functional (4) with respect to the initial geometrical descriptor and momentum. The deformation modules that we use here are combinations of constrained translations generator deformation modules (see Section 3.2.3) and then geometrical descriptors are sets of points. As a consequence, a natural and simple choice for the regularizing function $C_{1}$ is to set a maximum value $M$ for the norm of the points and define $C_{1}: a \in \mathcal{O} \mapsto \sum_{x \in a} \frac{1}{\|\left. x\right|^{2}-\left.M^{2}\right|^{2}}$ where the notation $\sum_{x \in a}$ means summation over all points in forming the geometrical descriptor $a$. The function $C_{2}$ is taken as the squared norm of the momentum. In all the experiments, the domain of the images is $\Omega=[-16,16] \times[-16,16]$ and is discretised using $256 \times 256$ pixels. The optimisation is performed via a gradient descent, and the gradient is computed with a forward and backward integration scheme as described in [13] (Section 6).

We present results of image reconstruction for two different types of operator $T$ : a 2-D tomography operator and a restricting operator (they are defined in the corresponding sections). The data are in most cases noisy data, and we will specify the noise level by PSNR, which is defined as

$$
\operatorname{SNR}(g)=10 \log _{10}\left(\frac{\left\|g_{0}-\overline{g_{0}}\right\|^{2}}{\|n-\bar{n}\|^{2}}\right)
$$

where $g_{0}$ is the noise-free part of the data and $n=g-g_{0}$ the noise-part and $\bar{x}$ denotes the mean of $x$. The PSNR is expressed in terms of decibel $\mathrm{dB}$.

In all the experiments, the initial geometrical are optimized but they are initialized with 'good' initial positions and then do not move a lot during the optimization.

\section{$5.2 \quad 2-\mathrm{D}$ tomography operator}

\subsubsection{2-D tomography operator}

In this example the forward operator $T$ is the 2-D Ray transform defined by, for $I \in$ $L^{2}(\Omega, \mathbb{R})$,

$$
T(I):(w, x) \in S^{1} \times \mathbb{R} \mapsto \int_{s \in \mathbb{R}, x+s w \in \Omega} I(x+s w) \mathrm{d} s
$$

where $S^{1}$ is the unit circle. In the discretised setting, we specify the angles (discretisation of $S^{1}$ ) and the number of lines per angle (discretization of a bounded interval of $\mathbb{R}$ ). In the following examples, we use 100 angles uniformly distributed in $[0, \pi]$ and 724 angles per line. 

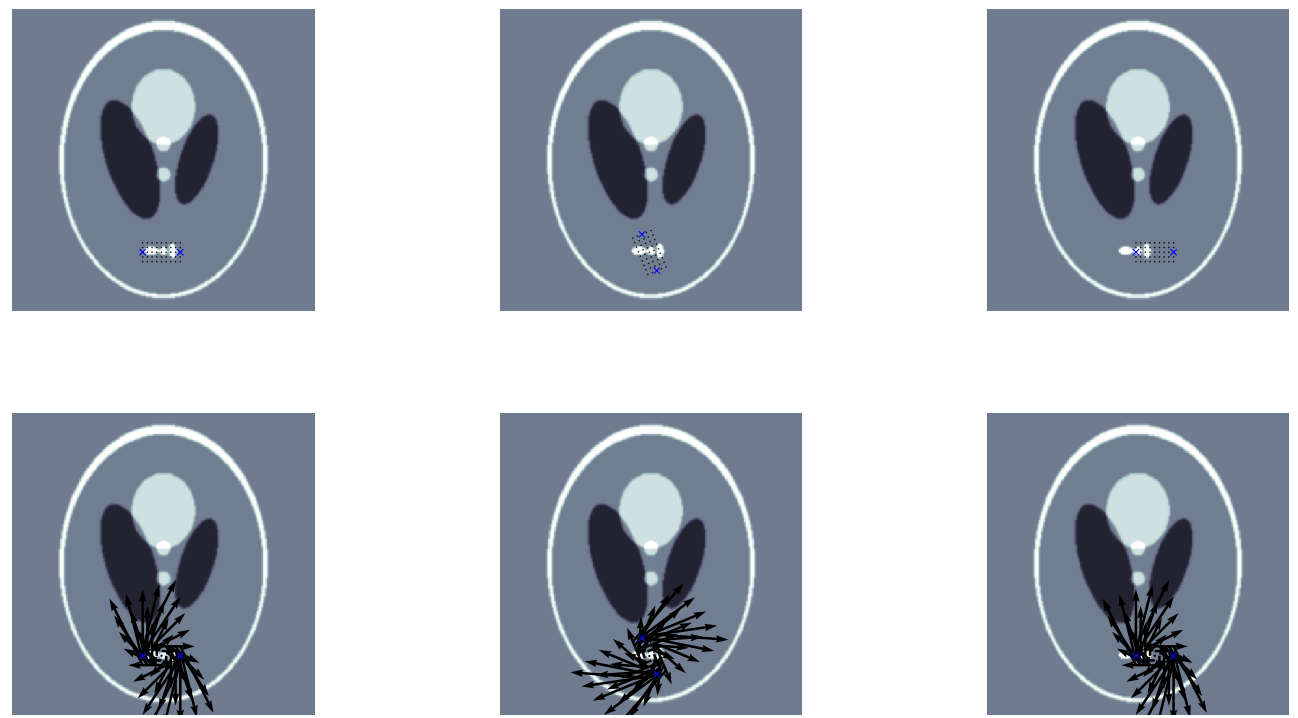

Figure 7: Examples of points $f(o)$ (first row, black points) and vectors $g(o)$ (second row, black vectors) generated by the deformation module presented in Section 5.2.2 for 3 different geometrical descriptors (in blue).

\subsubsection{Local rotation}

We will first consider the same noise-free data as in Figure 1 for which unconstrained deformation frameworks do not give satisfying results. In order to obtain a better reconstructed image via constrained deformations, the prior to incorporate in the deformation model is that there should be an anisotropic rotation acting in the area of the small white structure. We present here an easy way to build a constrained translations generator deformation module corresponding to this prior. We set the kernel size $\sigma=0.5, \mathcal{O}=\mathbb{R}^{2} \times \mathbb{R}^{2}$ (geometrical descriptors are formed of two points) and we define $f$ by associating, to each geometrical descriptor $o=\left(o_{1}, o_{2}\right)$, points regularly spaced by a distance $\sigma$ in a rectangle grid of axis $o_{1}-o_{2}$ and its orthogonal, see Figure 7. Then we define the function $g$ so that the vector associated to $f_{i}(o)$ is $g_{i}(o)=R_{\frac{o_{1}+o_{2}}{2}, \frac{\pi}{2}}\left(f_{i}(o)\right)$ where $R_{\frac{o_{1}+o_{2}}{2}, \frac{\pi}{2}}$ is the infinitesimal rotation (angle $\frac{\pi}{2}$ ) centred at $\frac{o_{1}+o_{2}}{2}$ (see Figure 7 ).

In figure we show the result of the indirect registration using this deformation module. We can see here that as we only allow the vector field at each time to be a local rotation, the desired deformations occurs. Then, if necessary, one could for study the estimated parameters of this deformation (given by the initial momentum).

\subsubsection{Local rotation and additional deformation}

Let us now consider the case where the ground truth is the image in Figure $9 \mathrm{c}$ and data are noisy (see Figure $9 \mathrm{~b}, S N R=9.8$ ). In this case there are additional differences between the template and the ground truth. Let us suppose that the only prior that we have about the form of the deformation is that there are a "pushing-forces" acting (this can for instance model a growth) and that the area on which they act can be modelled via a Gaussian kernel. The easiest way to model this is via translations. We then build two deformation modules, each one generating one local translation (the kernel sizes are respectively 1 and 


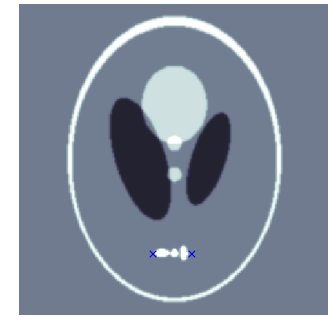

(a) Template.

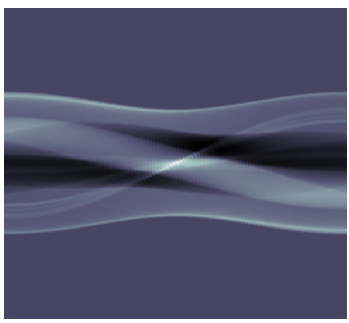

(b) Data.

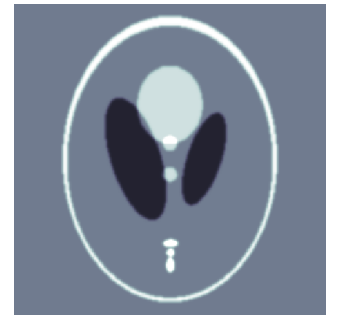

(c) Ground truth.

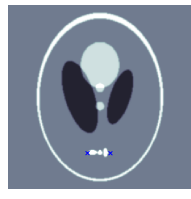

(d) $t=0$.

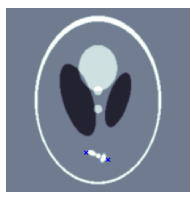

(e) $t=0.25$.

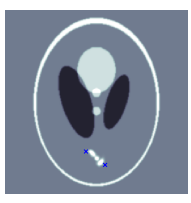

(f) $t=0.5$.

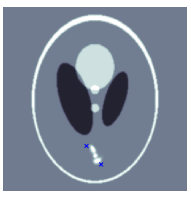

(g) $t=0.75$.

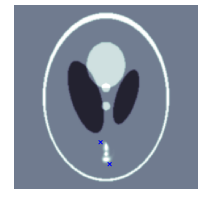

(h) $t=1$.

Figure 8: Result of constrained indirect matching. Template $I_{0}$ in fig. 8a matched against data $g$ in fig. 8b obtained from ground truth in fig. 8c (forward operator : Ray transform with 100 angles uniformly distributed 0 and $\pi$ ) with the deformation module presented in Section 5.2.2. Second row : image trajectory $\varphi_{t}^{\zeta_{o}(h)} \cdot I_{0}$, the reconstructed image is then in Figure 8h. The blue crosses are the geometrical descriptors (their initialisation are in fig. 8a).

2 and are supposed to be known). We also use the previous deformation module generating a local and anisotropic rotation. Then we combine these three deformation modules (see Section 3.3). The result of the indirect registration using this compound deformation module is presented in Figure 9. As previously, the adapted rotation deformation is estimated by the gradient descent, and simultaneously the two translations "push" in the good direction to lead to a satisfying image reconstruction (fig. 9h).

This examples illustrates how one can easily complexify pre-existing deformation constraints (modelled by a given deformation module) by building new deformation modules and combining them with the pre-existing deformation module.

\subsection{Reconstruction from a partial observation}

We present now an example where the operator is a restriction operator which means that we only observe a small area of the whole image. This area will be a rectangle and then defined by its extremal points. This example illustrates how a prior knowledge about a 'large-scale motion' can allow to reconstruct an image from a 'small-scale observation'. The template, ground truth image and data are presented in Figure 12 (the observation window for the data is $[-5,5] \times[-5,5]$ and the SNR is 3$)$. We suppose that we know that only two types of motions can happen here: an horizontal compressing motion (see Figure 10a) and a shearing motion (moving horizontally, see Figure 10b). A simple way to build a constrained translations generator deformation module generating compressing (resp. shearing) vector field is to set $\mathcal{O}=\mathbb{R}^{2} \times \mathbb{R}^{2}, f=I d_{\mathcal{O}}$ and $g: o=\left(o_{1}, o_{2}\right) \mapsto\left(o_{1}-o_{2}, o_{2}-o_{1}\right)$ (resp. $g: o=\left(o_{1}, o_{2}\right) \mapsto\left(\left(o_{1}-o_{2}\right)^{T},\left(o_{2}-o_{1}\right)^{T}\right)$ with $x^{T} \doteq\left(x_{2},-x_{1}\right)$ for $\left.x=\left(x_{1}, x_{2}\right) \in \mathbb{R}^{2}\right)$. See Figure 11 for illustrations of this construction.

The result of the indirect registration with the combination of these two deformation 


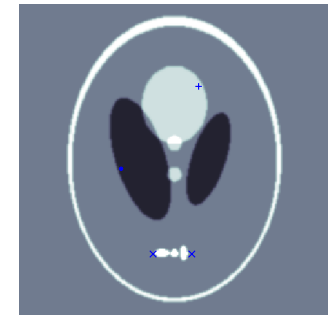

(a) Template.

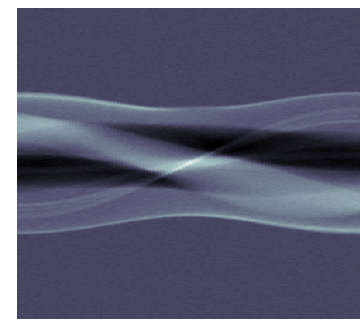

(b) Noisy data.

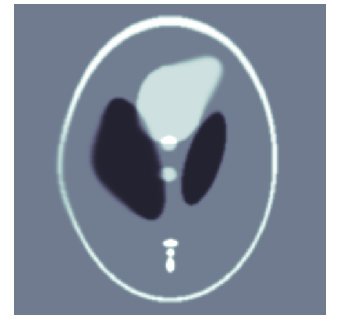

(c) Ground truth.

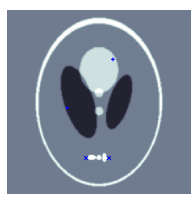

(d) $t=0$.

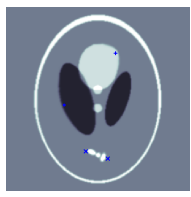

(e) $t=0.25$.

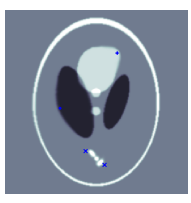

(f) $t=0.5$.

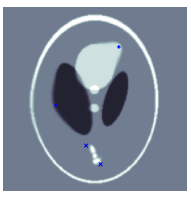

(g) $t=0.75$.

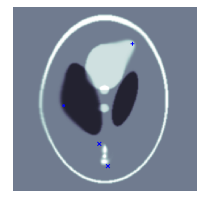

(h) $t=1$.

Figure 9: Result of constrained indirect matching. Template $I_{0}$ in fig. 9a matched against noisy data $g$ in fig. $9 \mathrm{~b}$ obtained from ground truth in fig. 9c (forward operator : Ray transform with 100 angles uniformly distributed 0 and $\pi$ ) with the deformation module presented in Section 5.2.3. Second row : image trajectory $\varphi_{t}^{\zeta_{o}(h)} \cdot I_{0}$, the reconstructed image is then in fig. 9h. The geometrical descriptors are in blue (crosses for the anisotropic rotation, plus for the translation with $\sigma=2$ and dot for the translation with $\sigma=1$ ).

modules is presented in Figure 12. The image is well reconstructed and then allow to understand how the whole image is deformed even if only a small part is observed.

\section{Conclusion}

We have presented a framework to reconstruct images as transformations of a known template image via constrained deformations. We showed that this is a well-defined regularization method, and illustrated that it allows to perform good reconstruction on 2examples with noisy data of various natures. The deformations are constrained via constrained translations generator deformation modules which are a particular category of deformation modules that is easy to use and can produce a wide variety of deformations.

The method that we present supposes that the template image is known but in practice it can be necessary to estimate it as well. In order to do so we will develop an iterative scheme where this image and the deformation are alternatively optimized.

In all the numerical examples, we supposed that the appropriate deformation modules we perfectly known. In particular we suppose that the Gaussian kernel is an appropriate localizing function and that its kernel-size is known. This will in general not be the case with real data and we currently work on automatically defining deformation modules adapted to given data.

\section{Acknowledgements}

The work by Barbara Gris was supported by the Swedish Foundation for Strategic Research grant AM13-0049. 


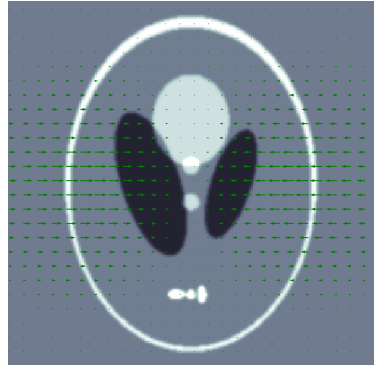

(a) Compressing motion.

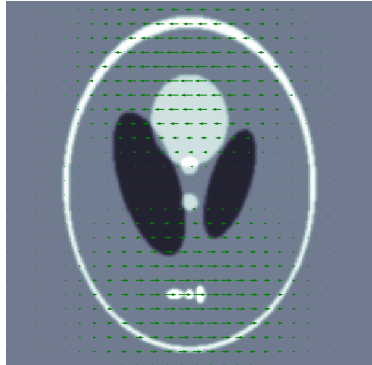

(b) Shearing motion.

Figure 10: Motions modelled in Section 5.3
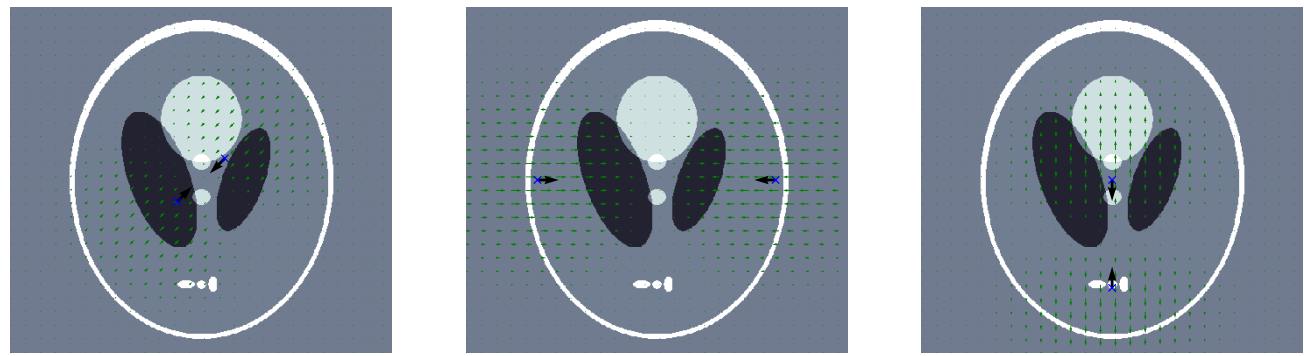

(a) Vector fields generated by deformation module generating compressing-dilating field. Geometrical descriptors are crosses.
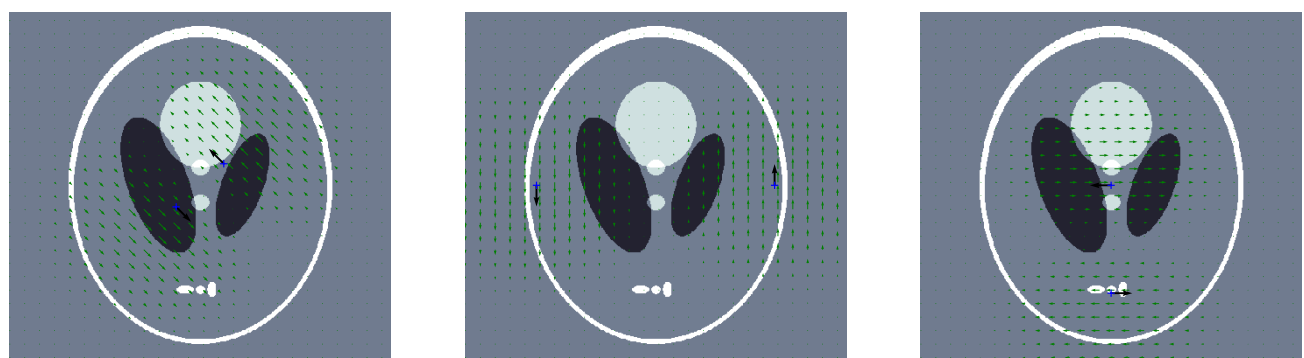

(b) Vector fields generated by deformation module generating shearing fields. Geometrical descriptors are pluses.

Figure 11: Examples of vector fields generated by the two deformation modules defined in Sec5.3 ( $\sigma=8$ for both). The geometrical descriptors $o$ are plotted in blue, vectors $g(o)$ are in black and the vector fields in green (the scalar controls are not represented). 


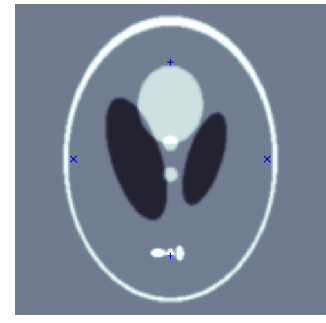

(a) Template.

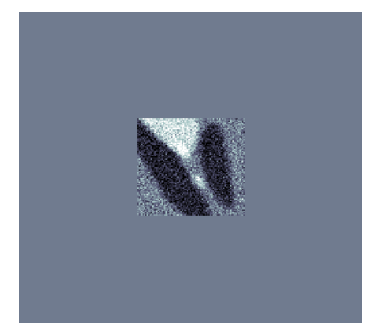

(b) Noisy data.

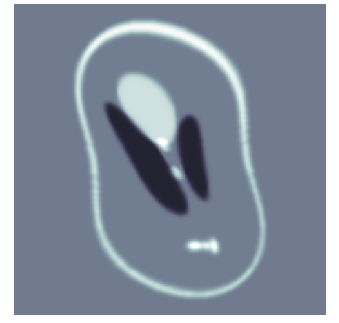

(c) Ground truth.

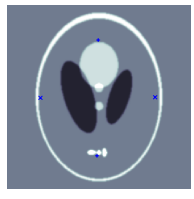

(d) $t=0$.

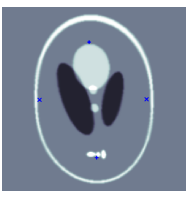

(e) $t=0.25$.

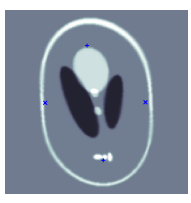

(f) $t=0.5$.

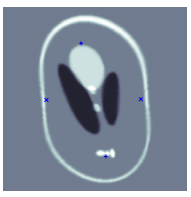

(g) $t=0.75$.

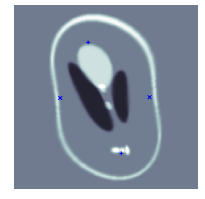

(h) $t=1$.

Figure 12: Result of constrained indirect matching. Template $I_{0}$ in fig. 12a matched against noisy data $g$ in fig. $12 \mathrm{~b}$ obtained from ground truth in fig. 12c (forward operator: restricting operator) with the deformation module presented in Section 5.3. Second row: image trajectory $\varphi_{t}^{\zeta_{o}(h)} \cdot I_{0}$, the reconstructed image is then in fig. $12 \mathrm{~h}$. The geometrical descriptors are in blue (crosses for the compressing-field module and pluses for the shearing-field module.)

\section{References}

[1] Y. Amit, U. Grenander, and M. Piccioni. Structural image restoration through deformable templates. Journal of the American Statistical Association, 86(414):376-387, 1991.

[2] S. Arguillere. Géométrie sous-riemannienne en dimension infinie et applications à l'analyse mathématique des formes. PhD thesis, Paris 6, 2014.

[3] S. Arguillere, E. Trélat, A. Trouvé, and L. Younes. Shape deformation analysis from the optimal control viewpoint. Journal de mathématiques pures et appliquées, 2015.

[4] V. Arsigny, O. Commowick, N. Ayache, and X. Pennec. A fast and log-euclidean polyaffine framework for locally linear registration. Journal of Mathematical Imaging and Vision, 33(2):222-238, 2009.

[5] V. Arsigny, X. Pennec, and N. Ayache. Polyrigid and polyaffine transformations: a novel geometrical tool to deal with non-rigid deformations-application to the registration of histological slices. Medical image analysis, 9(6):507-523, 2005.

[6] M. F. Beg, M. I. Miller, A. Trouvé, and L. Younes. Computing large deformation metric mappings via geodesic flows of diffeomorphisms. International journal of computer vision, 61(2):139-157, 2005.

[7] M. Blume, A. Martinez-Moller, A. Keil, N. Navab, and M. Rafecas. Joint reconstruction of image and motion in gated positron emission tomography. IEEE transactions on medical imaging, 29(11):1892-1906, 2010. 
[8] M. Bruveris and D. D. Holm. Geometry of image registration: The diffeomorphism group and momentum maps. In Geometry, Mechanics, and Dynamics, pages 19-56. Springer, 2015.

[9] M. Burger, H. Dirks, L. Frerking, A. Hauptmann, T. Helin, and S. Siltanen. A variational reconstruction method for undersampled dynamic x-ray tomography based on physical motion models. Inverse Problems, 33(12):124008, 2017.

[10] C. Chen and O. Öktem. Indirect image registration with large diffeomorphic deformations. SIAM Journal of Imaging Sciences, 11(1):575-617, 2018.

[11] J. Ehrhardt, R. Werner, D. Säring, T. Frenzel, W. Lu, D. Low, and H. Handels. An optical flow based method for improved reconstruction of $4 \mathrm{~d}$ ct data sets acquired during free breathing. Medical Physics, 34(2):711-721, 2007.

[12] U. Grenander, A. Srivastava, and S. Saini. A pattern-theoretic characterization of biological growth. Medical Imaging, IEEE Transactions on, 26(5):648-659, 2007.

[13] B. Gris. Modular approach on shape spaces, Sub-Riemannian geometry and computational anatomy. PhD thesis, Université Paris-Saclay, 2016.

[14] B. Gris, S. Durrleman, and A. Trouvé. A sub-riemannian modular framework for diffeomorphism based analysis of shape ensembles. 2016.

[15] B. N. Hahn. Motion estimation and compensation strategies in dynamic computerized tomography. Sensing and Imaging, 18(1):10, 2017.

[16] J. Hinkle, M. Szegedi, B. Wang, B. Salter, and S. Joshi. 4d ct image reconstruction with diffeomorphic motion model. Medical image analysis, 16(6):1307-1316, 2012.

[17] A. Isola, A. Ziegler, T. Koehler, W. Niessen, and M. Grass. Motion-compensated iterative cone-beam ct image reconstruction with adapted blobs as basis functions. Physics in Medicine 63 Biology, 53(23):6777, 2008.

[18] J. Karlsson and A. Ringh. Generalized sinkhorn iterations for regularizing inverse problems using optimal mass transport. SIAM Journal on Imaging Sciences, 10(4):1935-1962, 2017.

[19] A. Katsevich. An accurate approximate algorithm for motion compensation in twodimensional tomography. Inverse Problems, 26(6):065007, 2010.

[20] W. Lu and T. R. Mackie. Tomographic motion detection and correction directly in sinogram space. Physics in Medicine $\mathscr{E}$ Biology, 47(8):1267, 2002.

[21] B. A. Mair, D. R. Gilland, and J. Sun. Estimation of images and nonrigid deformations in gated emission ct. IEEE transactions on Medical Imaging, 25(9):1130-1144, 2006.

[22] K. McLeod, M. Sermesant, P. Beerbaum, and X. Pennec. Spatio-temporal tensor decomposition of a polyaffine motion model for a better analysis of pathological left ventricular dynamics. IEEE transactions on medical imaging, 34(7):1562-1575, 2015.

[23] N. Portman. The modelling of biological growth: A pattern theoretic approach. 2010.

[24] M. Reyes, G. Malandain, P. M. Koulibaly, M. A. González-Ballester, and J. Darcourt. Model-based respiratory motion compensation for emission tomography image reconstruction. Physics in Medicine \& Biology, 52(12):3579, 2007. 
[25] S. Rit, J. Wolthaus, M. van Herk, and J.-J. Sonke. On-the-fly motion-compensated cone-beam ct using an a priori motion model. In International Conference on Medical Image Computing and Computer-Assisted Intervention, pages 729-736. Springer, 2008 .

[26] C. J. Ritchie, J. Hsieh, M. F. Gard, J. D. Godwin, Y. Kim, and C. R. Crawford. Predictive respiratory gating: a new method to reduce motion artifacts on ct scans. Radiology, 190(3):847-852, 1994.

[27] M.-M. Rohé, N. Duchateau, M. Sermesant, and X. Pennec. Combination of polyaffine transformations and supervised learning for the automatic diagnosis of lv infarct. In International Workshop on Statistical Atlases and Computational Models of the Heart, pages 190-198. Springer, 2015.

[28] C. Seiler, X. Pennec, and M. Reyes. Capturing the multiscale anatomical shape variability with polyaffine transformation trees. Medical image analysis, 16(7):13711384, 2012.

[29] A. Srivastava, S. Saini, Z. Ding, and U. Grenander. Maximum-likelihood estimation of biological growth variables. In Energy Minimization Methods in Computer Vision and Pattern Recognition, pages 107-118. Springer, 2005.

[30] G. Van Eyndhoven, J. Sijbers, and J. Batenburg. Combined motion estimation and reconstruction in tomography. In European Conference on Computer Vision, pages 12-21. Springer, 2012.

[31] L. Younes. Constrained diffeomorphic shape evolution. Foundations of Computational Mathematics, 12(3):295-325, 2012. 